\title{
Salvage high-dose-rate brachytherapy for histologically confirmed macroscopic local relapsed prostate cancer after radical prostatectomy
}

\author{
David Buchser, MD', Alfonso Gomez-Iturriaga, MD, PhD', J. Ignacio Rodriguez Melcon, MD, PhD², \\ Francisco Casquero, MD, PhDl, Roberto Llarena, MD, PhD³, Jon Cacicedo, MD, PhDl, Pedro Bilbao, MD, PhD' \\ IHospital Universitario Cruces/Biocruces Health Research Institute, Radiation Oncology, Barakaldo, ${ }^{2}$ Hospital Universitario Negrin, Las Palmas, \\ Canary Islands, ${ }^{3}$ Hospital Universitario Cruces, Urology, Barakaldo, Spain
}

\begin{abstract}
Purpose: To evaluate the feasibility of the use of real-time magnetic resonance imaging (MRI)-transrectal ultrasound (TRUS) fusion guided high-dose-rate brachytherapy (HDR-BT) +/- external beam radiation therapy (EBRT) in patients with histologically-proven local relapse after radical prostatectomy.

Material and methods: We retrospectively reviewed 13 patients treated with real-time MRI-TRUS fusion HDR-BT for a local relapse of prostate cancer after radical surgery. All patients underwent multiparametric magnetic resonance imaging (mpMRI) to confirm the presence of macroscopic lesions in prostate bed, and choline positron emission tomography/computed tomography (PET/CT) to rule out nodal or distant metastases. Local failure was confirmed by transrectal biopsy. Patients without previous EBRT received 1 fraction of 15 Gy with HDR-BT plus hypofractionated EBRT (37.5 Gy in 15 fractions). Two patients received 2 fractions of 12 Gy with HDR-BT without EBRT. Follow-up visits were at 1, 3, 6 months, and every 6 months thereafter.

Results: After a median follow-up of 7 months, all patients showed an appropriate biochemical response. Median prostate-specific antigen (PSA) levels before treatment, 1 month, and 6 months after HDR-BT were $2.62 \mathrm{ng} / \mathrm{ml}$ (range: $1.55-9.61), 0.97 \mathrm{ng} / \mathrm{ml}$ (range: $0.12-3.14), 0.23 \mathrm{ng} / \mathrm{ml}$ (range: $0.1-0.74$ ), respectively. Five patients (42\%) experienced acute grade $1 \mathrm{GU}$ toxicity and 1 patient $(8 \%)$ suffered from grade $2 \mathrm{GU}$ toxicity. Regarding gastrointestinal (GI) toxicity, 5 patients referred grade 1 acute toxicity and 1 grade 2 (proctitis). No late toxicity has been observed so far.

Conclusions: MRI-TRUS fusion guided salvage HDR-BT +/- EBRT is a feasible procedure for patients with local macroscopic relapse in tumor bed after radical prostatectomy. Exquisite selection of patients through mpMRI and choline PET/CT is crucial to avoid overtreatment. A larger number of patients and longer follow-up are required in order to draw more solid conclusions regarding the effectiveness of this strategy.
\end{abstract}

Key words: high-dose-rate, local relapse, prostate cancer, salvage.

\section{Purpose}

Nearly $30 \%$ of patients undergoing radical prostatectomy experience recurrence within 10 years [1] and there is an increased risk when adverse pathologic features (i.e. positive surgical margins, seminal vesicle invasion, extraprostatic extension, and higher Gleason score) appear. The treatment failure pattern after prostatectomy is predominantly local [2]. In this setting, external beam radiation therapy (EBRT) with or without androgen deprivation therapy (ADT) is the most common salvage treatment [3]. Besides, there is retrospective evidence supporting a benefit from salvage EBRT for a rising prostate-specific antigen (PSA) in patients without evidence of distant metastases $[4,5,6,7]$.
Radiation doses for salvage therapy range from 64 to 72 Gy and higher doses may be needed for biopsy proven macroscopic disease [8]. Furthermore, dose escalation protocols achieve better tumor control rates $[9,10]$. Unfortunately, these treatments are associated with significant genitourinary (GU) toxicity, mainly due to the need to include the bladder neck and the vesicourethral anastomosis in the treatment field.

High-dose-rate brachytherapy (HDR-BT) is a highly conformal method of radiation dose escalation for prostate cancer with potential benefits over conventional EBRT, accurately adjusting the isodoses to the target while keeping adjacent organs such as the urethra and rectum within tolerance [11]. The high precision of BT allows to ad- 
minister extreme hypofractionation schedules exploiting the low $\alpha / \beta$ ratio of prostate cancer, hence, the biological radiation dose delivered is significantly greater than that achieved with EBRT [12].

On the other hand, recent advances in multiparametric magnetic resonance imaging (mpMRI) and choline positron emission tomography/computed tomography (PET/CT) have markedly improved detection of local and distant recurrence following therapy [13]. Therefore, choline PET/CT and mpMRI have an important role in the detection and location of recurrences at this moment, allowing a better selection of candidates for local salvage treatment [14].

There are very few reported series of patients undergoing BT for local relapse after radical prostatectomy and only two of them used HDR-BT.

This is a preliminary study designed to evaluate the feasibility of the use of real-time magnetic resonance imaging (MRI)-transrectal ultrasound (TRUS) fusion guided HDR-BT + / - EBRT in patients with histologically-proven clinical local relapse after radical prostatectomy.

Table 1. Patient characteristics

\begin{tabular}{|c|c|c|}
\hline Factor & Median & Range \\
\hline Age (years) & 71.5 & $66-78$ \\
\hline iPSA (ng/ml) & 5.7 & $4.41-25.7$ \\
\hline Time to salvage treatment (months) & 121 & $52-192$ \\
\hline ADC value & 0.735 & $0.53-0.93$ \\
\hline PSA pre-BT (ng/ml) & 2.71 & $1.55-13.93$ \\
\hline Gleason score & $N$ & \\
\hline 5 & 1 & \\
\hline 6 & 5 & \\
\hline 7 & 2 & \\
\hline 8 & 5 & \\
\hline T stage & $N$ & \\
\hline $1 c$ & 1 & \\
\hline $2 a$ & 2 & \\
\hline $2 b$ & 5 & \\
\hline $2 c$ & 2 & \\
\hline $3 a$ & 3 & \\
\hline Surgery & N & \\
\hline Open prostatectomy & 12 & \\
\hline Laparoscopic prostatectomy & 1 & \\
\hline Margins & $N$ & \\
\hline $\mathrm{Neg}$ & 5 & \\
\hline Pos & 8 & \\
\hline
\end{tabular}

$A D C$ - apparent diffusion coefficient, BT - brachytherapy, iPSA - initial PSA PSA - prostate-specific antigen

\section{Material and methods}

We retrospectively reviewed 13 patients treated with MRI/TRUS fusion HDR-BT from October 2014 to March 2016, for a local relapse of prostate adenocarcinoma after radical surgery. All patients underwent mpMRI to confirm the presence of macroscopic lesions in prostate bed and choline PET/CT to rule out nodal or distant metastases. Local failure was confirmed by transrectal biopsy. Patient characteristics and prior treatment for prostate cancer are summarized in Table 1. Acute and late toxicities were classified and graded according to the Common Terminology Criteria for Adverse Events version 4.0 definition.

Magnetic resonance imaging technique has been previously reported $[15,16]$. In brief, MRI is performed using a $1.5 \mathrm{~T}$ Achieva scanner (Philips Electronics, Eindhoven, The Netherlands), and a body multichannel antenna (Cardiac Sense-MS). Axial T1 sequences of the entire pelvis from the iliac crest to pubic symphysis and T2 axial volumetric sequence (VISTA) as a guide for planning brachytherapy were performed. For the functional study, diffusion (DWI) sequences were performed. The apparent diffusion coefficient (ADC) values were obtained from (DWI) sequences, and finally a dynamic fast field echo (FFE) T1 volumetric sequence was performed. From the resulting image data, various curves of perfusion were analyzed to detect and localize the tumor. Radiologists experienced in uro-radiology evaluated all magnetic resonance studies (Figure 1).

Patients without previous adjuvant or salvage radiotherapy received combined MRI-TRUS fusion HDR-BT (1 fraction of $15 \mathrm{~Gy}$ ), followed by 2-4 weeks of hypofractionated EBRT (37.5 Gy in 15 fractions) to the prostate bed. Two patients received two fractions of $12 \mathrm{~Gy}$, one week apart, with HDR-BT without EBRT because they had already undergone irradiation to the prostate bed as salvage therapy in the setting of an early biochemical relapse after radical prostatectomy. The MRI-TRUS fusion technique has been previously reported $[15,16]$. Briefly, the T2 axial volumetric sequence (VISTA) is imported directly from the picture archiving and communication systems (PACS), and sent to the Oncentra ${ }^{\circledR}$ Prostate v. 4.0 software (Nucletron, an Elekta company, Elekta AB, Stockholm, Sweden). Magnetic resonance images are reconstructed and segmented. Target volumes, including macroscopic relapse, urethra, bladder, and rectum were contoured. To delineate the macroscopic relapse, information from T2, ADC map, and DCE sequences were used.

A transrectal sagittal volumetric ultrasound image is immediately acquired with images obtained every 0.5 degrees. A rapid reconstruction algorithm converts the series of $2 \mathrm{D}$ images into a $3 \mathrm{D}$ volume, which is then displayed in axial, sagittal, and coronal views and transferred to the fusion module. The MRI and the real-time ultrasound examination are displayed on a split-screen with the possibility of overlaying the images live in one image. A graphical user interface is used for rigid manual registration of the ultrasound and MRI. This interface allows for displacements in three dimensions as well as rotations, until both images are correctly superimposed. The contoured structures are transferred to the ultra- 

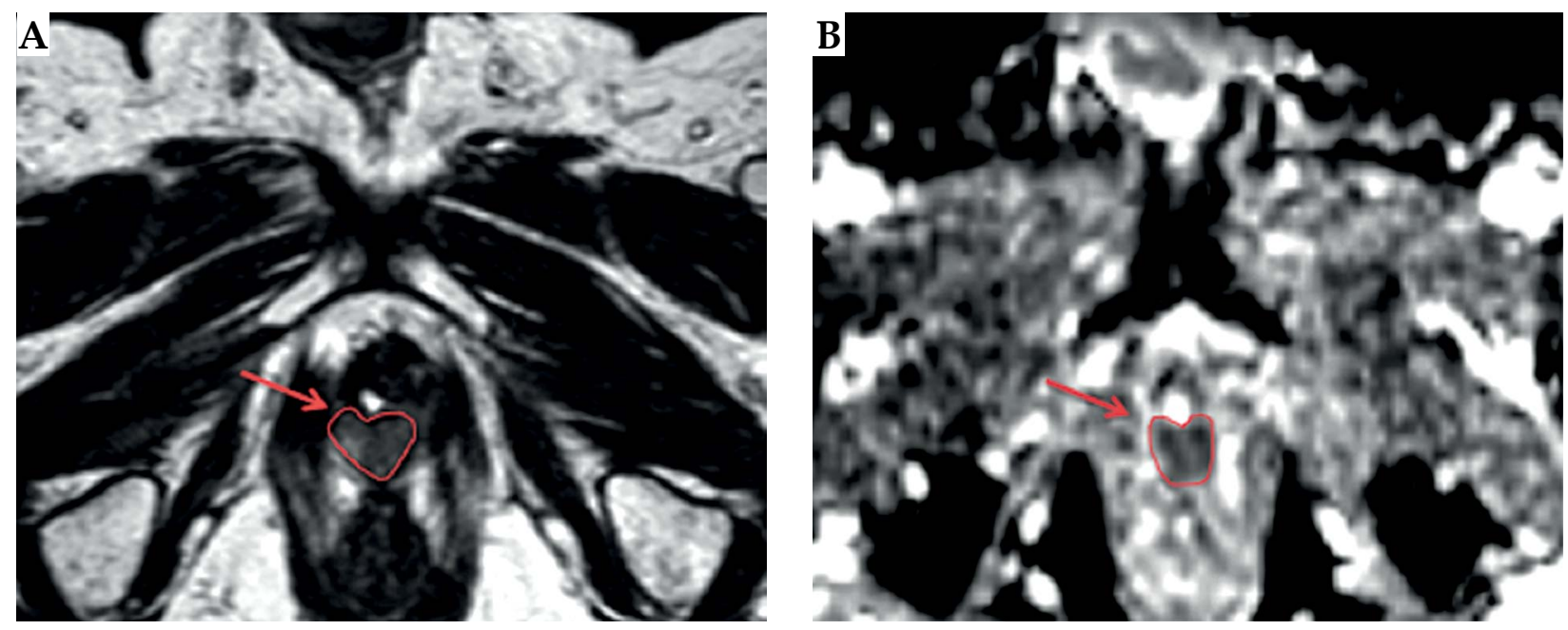

Fig. 1. Diagnostic multiparametric magnetic resonance imaging. Red contour and arrows indicate the local relapse: left image, T2 sequence; right image, ADC map
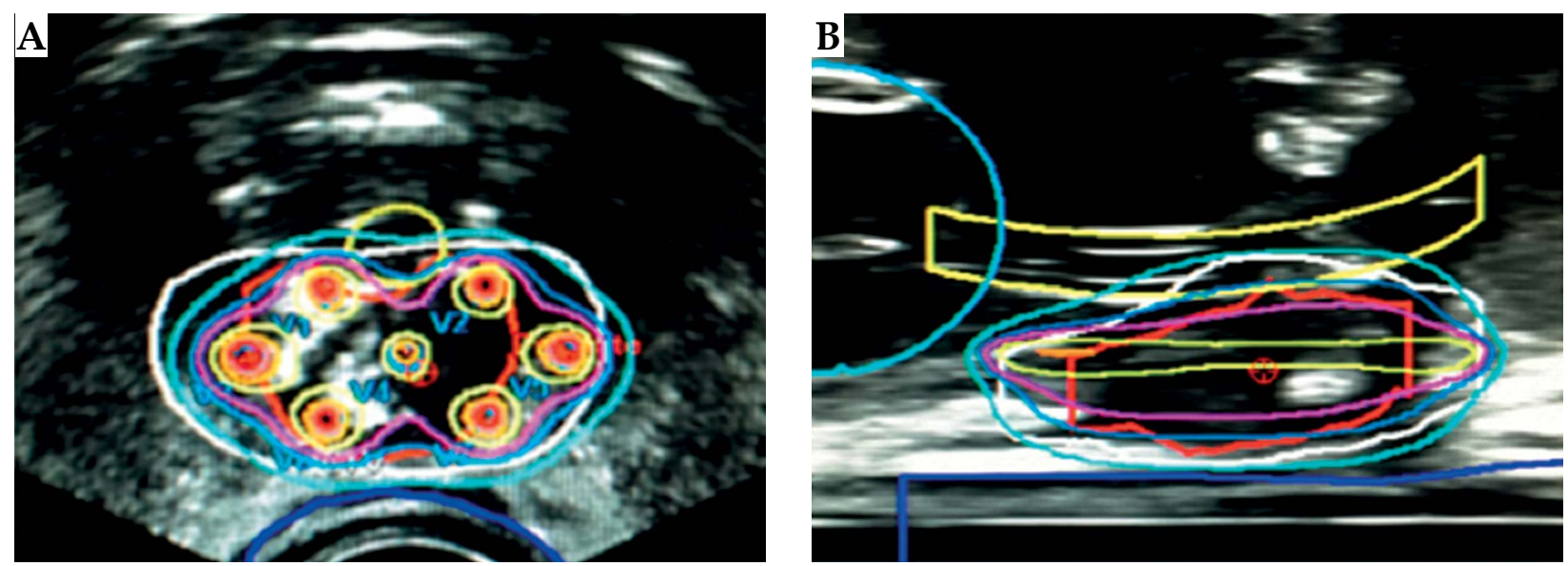

Fig. 2. Local relapse seen on transrectal ultrasound image. High-dose-rate brachytherapy plan. Right - axial view, left - sagittal view

sound dataset. These contours may be slightly modified, until a perfect match with the US images is achieved. A clinical target volume (CTV) was created, $5 \mathrm{~mm}$ margin in all directions with the exception of $1 \mathrm{~mm}$ margin when necessary to avoid overlapping with organs at risk such as the rectum or the urethra. A peripheral implant of the lesion was performed to ensure enough marginal dose coverage of the target volumes (Figure 2). For the EBRT phase, we followed the RTOG contouring guidelines for the delineation of the prostate bed [17].

Descriptive statistics (means and standard deviations) were calculated to summarize the clinical characteristics of the 13 patients and dosimetric parameters for each treatment plan. Follow-up visits were at 1, 3, 6 months, and every 6 months thereafter.

\section{Results}

Eleven patients received a single BT fraction of $15 \mathrm{~Gy}$, followed by $37.5 \mathrm{~Gy}$ in 15 fractions delivered with EBRT. Two patients received two fractions of 12 Gy HDR-BT.
The initial tumor characteristics are summarized in Table 1 . Median age was 71.5 years (range: $66-78$ ). Median PSA pre-BT was $2.71 \mathrm{ng} / \mathrm{ml}$ (range: $1.84-13.93 \mathrm{ng} / \mathrm{ml}$ ) and median PSA doubling time was 7.8 months (range: 1.42113.03 months). Median time from initial surgery to salvage treatment was 121 months (range: 52-192 months).

Most local recurrences occurred at the perianastomotic site $(10 ; 77 \%)$, followed by the left seminal vesicle $(2 ; 15 \%)$, and pararectal site $(1 ; 8 \%)$.

\section{Treatment characteristics}

The median volume of the local relapse on mpMRI was $3.34 \mathrm{cc}$ (range: 1.98-6.76 cc). The median number of needles used in the brachytherapy procedure was 7 (range: 5-9). Median CTV $\mathrm{D}_{90}$ and $\mathrm{V}_{100}$ were $112.68 \%$ (range: 103.33-121.32\%) and 97.42\% (range: 93.6-99.26\%), respectively. The complete dosimetric characteristics of the HDR plans are summarized in Table 2.

After a median follow-up of 7 months (range: 4-15) all of our patients showed an appropriate biochemical response. Median PSA levels before treatment, 1 month, 
Table 2. Dosimetric parameters

\begin{tabular}{lcc} 
Dosimetric parameters & Mean & Range \\
\hline CTV & 112.68 & $103.33-121.32$ \\
\hline$D_{90}$ & 97.22 & $93.6-99.26$ \\
\hline$V_{100}$ & 35.98 & $20.32-61.68$ \\
\hline$V_{150}$ & 7.15 & $2.62-22.79$ \\
\hline$V_{200}$ & & \\
\hline Urethra & 90 & $40-115$ \\
\hline$D_{\max }$ & 69.6 & $30.39-83.78$ \\
\hline$D_{10}$ & 22.88 & $9.9-32.11$ \\
\hline$D_{1 c c}$ & & \\
\hline Rectum & 80 & $50-115$ \\
\hline$D_{\max }$ & 40.86 & $29.45-59.73$
\end{tabular}

CTV - clinical target volume, $D_{90}$ - minimal dose delivered to $90 \%$ of target volume, $V_{n}(150,200)$ - fractional volume of the organ that receives $n \%$ of the prescribed dose, Urethral $D_{\max }$ - maximum point dose inside the urethral volume $<115 \%$, Urethral $D_{10}$ - minimal dose delivered to $10 \%$ of urethra, Rectum $D_{1 c c}$ and $2 c c$-doses for the most exposed $1 \mathrm{cc}$ and $2 \mathrm{cc}$ volumes of the rectum

and 6 months after HDR-BT were $2.62 \mathrm{ng} / \mathrm{ml}$ (range: $1.55-9.61 \mathrm{ng} / \mathrm{ml}$ ), $0.97 \mathrm{ng} / \mathrm{ml}$ (range: $0.12-3.14 \mathrm{ng} / \mathrm{ml}$ ), $0.23 \mathrm{ng} / \mathrm{ml}$ (range: $0.1-0.74 \mathrm{ng} / \mathrm{ml}$ ), respectively. One patient experienced a slight increase of PSA in the $15^{\text {th }}$ month post-treatment, from $0.71 \mathrm{ng} / \mathrm{ml}$ to $1.18 \mathrm{ng} / \mathrm{ml}$.

Five patients $(42 \%)$ experienced acute grade $1 \mathrm{GU}$ toxicity (dysuria or nocturia), and only one $(8 \%)$ suffered from grade $2 \mathrm{GU}$ toxicity (nocturia). Regarding gastrointestinal (GI) toxicity, five patients referred grade 1 acute toxicity and one grade 2 (proctitis). None of our patients experienced severe acute GU or GI (i.e. grade 3 or greater). No late toxicity has been observed so far.

\section{Discussion}

Our study demonstrates that MRI-TRUS fusion guided salvage HDR-BT +/- EBRT is a feasible procedure for patients with local macroscopic relapse in tumor bed after radical prostatectomy. Rising PSA levels after radical prostatectomy do not differentiate local relapse from distant metastasis. A high PSA velocity and short PSA doubling time suggest the latter, whereas a slow increase in PSA levels is usually related with local relapse. Nonetheless, image techniques play a key role in location of the disease.

Traditional imaging studies (i.e. TRUS and abdominopelvic CT) have serious limitations. Normal TRUS after radical prostatectomy does not rule out local disease since it cannot detect small volume and isoechoic lesions. On the other hand, contrast enhanced abdominopelvic CT sensitivity is highly dependent on PSA levels and drops with PSA $<10 \mathrm{ng} / \mathrm{ml}$ when the chance of achieving local control with radical treatment is rather low.
Recent advances in mpMRI and choline PET/CT have markedly improved the detection of local and distant recurrences $[18,19]$. The mpMRI is particularly precise in detecting local relapses after radical surgery. Sensitivity and specificity in this setting for this technique range from $79-88 \%$ and $89-100 \%$, respectively [20,21,22]. Regarding choline PET/CT, even though its accuracy detecting local disease is lower than that of mpMRI, it has the advantage of detecting distant metastases, especially nodal involvement, with low PSA serum concentration $(>1 \mathrm{ng} / \mathrm{ml})$. This means that we are now able to precisely detect and locate recurrent disease and accurately design salvage treatments [19].

There is level 2a evidence supporting the use of salvage radiotherapy in patients with biochemical relapse after radical surgery [23]. Two large retrospective reviews $[6,7]$ found that PSA level prior to salvage treatment and radiation dose are independent factors significantly related with relapse free survival. This data suggest that early treatments and dose escalation protocols might translate into better tumor control rates $[9,24]$. There is raising concern about the potential toxicity associated with higher radiation doses to the prostate bed. In a retrospective single institution analysis by Goenka et al., the use of intensity-modulated radiotherapy (IMRT) was associated with a significant reduction in GI toxicity [25]. This benefit, however, was not observed in genitourinary toxicity, probably because the bladder neck and the vesicourethral anastomosis are necessarily included in the treatment field [10].

Our patients, nonetheless, differ slightly from these series because they have a histologically proven macroscopic relapse. Very few series differentiate macroscopic from microscopic disease in the setting of biochemical relapse after radical prostatectomy. A retrospective study by Mcdonald et al. [26], including 42 patients with a palpable local recurrence in the prostate bed treated with EBRT (median dose of $68 \mathrm{~Gy}$ ), found promising results. All 42 patients showed resolution of clinically detectable recurrence within 1 year after radiotherapy with acceptable toxicity levels. In the setting of a macroscopic relapse, it seems logical to increase the dose delivered to the gross nodule. It is important to note that most of the aforementioned studies relied on conventional imaging techniques to diagnose local relapse and rule out metastasis. In the case of the systematic reviews, they do not explicitly state if the analyzed studies employed mpMRI or PET/CT.

Considering the previous facts, we believe that real time MRI-TRUS fusion guided HDR-BT plus EBRT (IMRT/ image guided radiotherapy [IGRT]) has potential advantages over EBRT alone in a dose-escalated scheme for these patients. On one hand, by delivering much of the dose in one fraction with HDR-BT, we reduce dramatically the inter-fraction and intra-fraction organ motion variability. Secondly, the steep dose gradient inherent to brachytherapy combined with the anatomic resolution of MRI-TRUS fusion technique, and the possibility to set different dwell times and positions, allow us to achieve a highly accurate conformation and to spare the organs at risk (OAR) (including the bladder neck and vesicourethral anastomosis). In addition, the extreme hypofrac- 


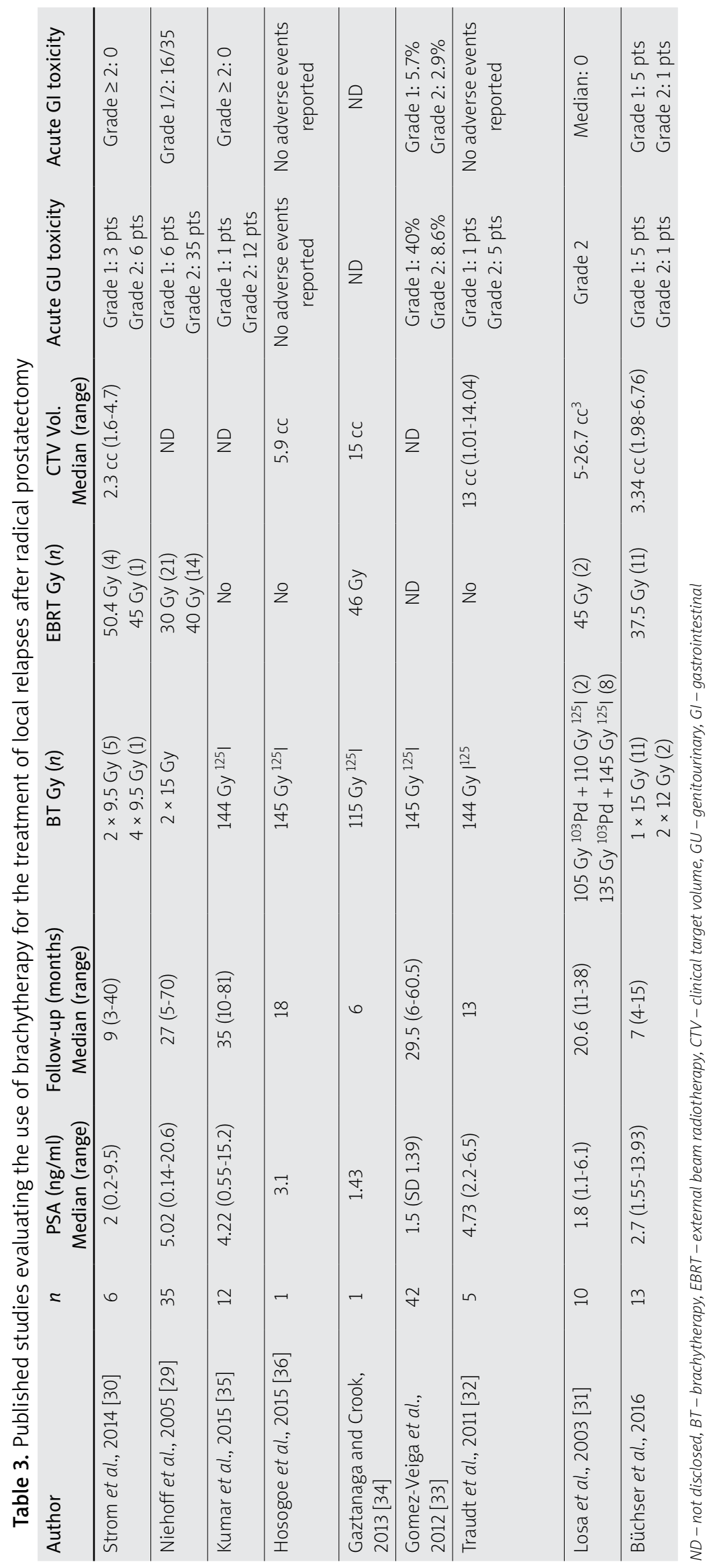


tionation achieved with HDR-BT, delivering large doses per fraction, takes advantage of the low $\alpha / \beta$ ratio of prostate cancer cells. Therefore, the biological equivalent radiation dose is significantly higher than that reached by EBRT-IMRT alone.

We decided to complete treatment with EBRT to a dose of $37.5 \mathrm{~Gy}$ administered in 15 fractions (2.5 Gy per fraction, $\mathrm{BED}_{1.5} 44.4 \mathrm{~Gy}$ ), to the prostate bed to account for potential microscopic disease outside the HDR-BT target.

Although there are some studies investigating the role of brachytherapy for local recurrences after prostate radiotherapy or brachytherapy $[27,28]$, very few series have reported results of salvage brachytherapy after radical prostatectomy and the number of patients included is rather small $[27,28,29,30,31,32,33,34,35,36]$. Table 3 shows a description of these series. Only two of them report results after HDR-BT. The largest HDR series published so far, by Niehoff et al. [29], included 35 patients treated with two 15 Gy HDR-BT sessions, 21 patients received complementary 30 Gy EBRT to the small pelvis (four-field box technique), whereas the remaining 14 received $40 \mathrm{~Gy}$. Mean follow-up for each group was 29 and 26 months, respectively. In the 30 Gy group, the PSA progression-free survival was 5/21 cases, and the mean time to PSA increase was 16 months (range: 6-42 months). Results for the 40 Gy group were similar, PSA progression-free survival was $6 / 14$ patients, and mean time interval to increase PSA was 10 months (range: 6-24 months). The differences are not statistically significant. No late or acute grade III/IV toxicity was recorded in either group (LENT SOMA, RTOG/EORTC).

More recently, Strom et al. [30] have published a series of 6 patients treated with HDR-BT with or without IMRT for biopsy-proven, recurrent prostate cancer post-prostatectomy. Five patients were treated with IMRT to 45-50.4 Gy in 25-28 fractions, followed by two 9.5 Gy HDR-BT sessions. One patient with a palpable, castrate-resistant recurrence following post-prostatectomy EBRT to 70 Gy (2 Gy per fraction) 8 years earlier, underwent salvage HDR-BT monotherapy consisting of 38 Gy delivered in four fractions over 3 days with a single implant. Median follow-up was 9 months (range: 3-40 months), at last follow-up, all patients had undetectable PSA levels. They report no severe toxicity.

With a shorter follow-up, our results are similar to those reported by the previous series. We observed slightly better outcomes regarding treatment related toxicities than that reported in both EBRT and brachytherapy series $[26,27,28]$, which could be explained by the use of the real time MRI/TRUS fusion protocol. All patients in our study showed an appropriate biochemical response; with PSA nadir of $0.73 \mathrm{ng} / \mathrm{ml}$ (range: $0.1-3.89 \mathrm{ng} / \mathrm{ml}$ ) at last follow-up visit, being the median pretreatment PSA $2.71 \mathrm{ng} / \mathrm{ml}$ (range: $1.84-13.93 \mathrm{ng} / \mathrm{ml}$ ). No grade 3 or 4 toxicity has been observed and only one patient presented grade 2 acute GU toxicity (nocturia) and another one presented grade 2 GI acute toxicity (proctitis).

Our series presents some advantages. Firstly, this is, to our knowledge, the first paper to report results with a real-time MRI/TRUS fusion HDR protocol for macroscopic local relapse of prostate adenocarcinoma. Secondly, thanks to the performance of mpMRI and choline PET/ $\mathrm{CT}$ in all our patients, we achieved a high level of accuracy in selecting patients who could benefit from local salvage treatment. Nonetheless, there are a few limitations in our study. It has the inherent biases of a retrospective analysis. Besides, this is a preliminary study to test the feasibility of the technique in our center, therefore, the short follow-up and the small number of patients do not allow to draw more solid conclusions. It is also hard to compare it with the different series results due to the heterogeneity of the BT schemes.

\section{Conclusions}

MRI-TRUS fusion guided salvage HDR-BT + / - EBRTIGRT is a feasible and safe procedure for patients with local macroscopic relapse in tumor bed after radical prostatectomy. Exquisite selection of patients through mpMRI and choline PET/CT is crucial to avoid overtreatment. A larger number of patients and longer follow-up are required in order to draw more solid conclusion regarding the safety and effectiveness of this strategy.

\section{Disclosure}

Authors report no conflict of interest.

\section{References}

1. Thompson IM, Valicenti RK, Albertsen P et al. Adjuvant and salvage radiotherapy after prostatectomy: AUA/ASTRO Guideline. J Urol 2013; 190: 441-449.

2. Swanson GP, Hussey MA, Tangen CM et al. SWOG 8794. Predominant treatment failure in postprostatectomy patients is local: analysis of patterns of treatment failure in SWOG 8794. J Clin Oncol 2007; 25: 2225-2229.

3. Punnen S, Cooperberg MR, D'Amico AV et al. Management of biochemical recurrence after primary treatment of prostate cancer: a systematic review of the literature. Eur Urol 2013; 64: 905-915.

4. Trock BJ, Han M, Freedland SJ et al. Prostate cancer-specific survival following salvage radiotherapy vs observation in men with biochemical recurrence after radical prostatectomy. JAMA 2008; 299: 2760-2769.

5. Boorjian SA, Karnes RJ, Crispen PL et al. Radiation therapy after radical prostatectomy: impact on metastasis and survival. J Urol 2009; 182: 2708-2714.

6. King CR. The timing of salvage radiotherapy after radical prostatectomy: a systematic review. Int J Radiat Oncol Biol Phys 2012; 84: 104-111.

7. Ohri N, Dicker AP, Trabulsi EJ et al. Can early implementation of salvage radiotherapy for prostate cancer improve the therapeutic ratio? A systematic review and regression metaanalysis with radiobiological modelling. Eur J Cancer 2008; 48: 837-844.

8. Prostate Cancer Guidelines.pdf [Internet]. [cited 2016 Jul 30]. Available from: https://www.nccn.org/professionals/physician_gls/pdf/prostate.pdf

9. De Meerleer G, Fonteyne V, Meersschout S et al. Salvage intensity-modulated radiotherapy for rising PSA after radical prostatectomy. Radiother Oncol 2008; 89: 205-213.

10. Ost P, Cozzarini C, Bossi A et al. Salvage radiotherapy: a plea for dose-escalation with intensity-modulated radiotherapy. Eur J Cancer 2012; 48: 1415-1416; author reply 1414. 
11. Morton G. The best method for dose escalation: Prostate brachytherapy. Can Urol Assoc J 2012; 6: 196-198.

12. Thiruthaneeswaran N, Hoskin PJ. High dose rate brachytherapy for prostate cancer: Standard of care and future direction. Cancer Radiothérapie 2016; 20: 66-72.

13. Notley M, Yu J, Fulcher AS et al. Pictorial review. Diagnosis of recurrent prostate cancer and its mimics at multiparametric prostate MRI. Br J Radiol 2015; 88: 20150362.

14. Cochet A, Kanoun S, Humbert O et al. Multimodality MRI and PET for restaging prostate cancer after biochemical failure of the treatment. Cancer Radiothérapie 2014; 18: 509-516 [Article in French].

15. Gomez-Iturriaga A, Crook J, Casquero F et al. Impact of intraoperative MRI/TRUS fusion on dosimetric parameters in CT3a prostate cancer patients treated with high-dose-rate real-time brachytherapy. J Contemp Brachytherapy 2014; 6: 154-160.

16. Gomez-Iturriaga A, Casquero F, Urresola A et al. Dose escalation to dominant intraprostatic lesions with MRI-transrectal ultrasound fusion High-Dose-Rate prostate brachytherapy. Prospective phase II trial. Radiother Oncol 2016; 119: 91-96.

17. Michalski JM, Lawton C, El Naqa I et al. Development of RTOG consensus guidelines for the definition of the clinical target volume for postoperative conformal radiation therapy for prostate cancer. Int J Radiat Oncol Biol Phys 2010; 76: 361-368.

18. Paparo F, Massollo M, Rollandi L et al. The clinical role of multimodality imaging in the detection of prostate cancer recurrence after radical prostatectomy and radiation therapy: past, present, and future. Ecancermedicalscience 2015; 9: 570.

19. Evangelista L, Zattoni F, Rossi E et al. Early detection of prostate cancer relapse by biochemistry and diagnostic imaging. Q J Nucl Med Mol Imaging 2015; 59: 359-373.

20. Cirillo S, Petracchini M, Scotti L et al. Endorectal magnetic resonance imaging at 1.5 Tesla to assess local recurrence following radical prostatectomy using T2-weighted and contrast-enhanced imaging. Eur Radiol 2009; 19: 761-769.

21. Casciani E, Polettini E, Carmenini E et al. Endorectal and dynamic contrast-enhanced MRI for detection of local recurrence after radical prostatectomy. AJR Am J Roentgenol 2008; 190: 1187-1192.

22. Panebianco V, Barchetti F, Sciarra A et al. Prostate cancer recurrence after radical prostatectomy: the role of 3-T diffusion imaging in multi-parametric magnetic resonance imaging. Eur Radiol 2013; 23: 1745-1752.

23. Stephenson AJ, Scardino PT, Kattan MW et al. Predicting the outcome of salvage radiation therapy for recurrent prostate cancer after radical prostatectomy. J Clin Oncol 2007; 25: 2035-2041.

24. Ost P, Lumen N, Goessaert AS et al. High-dose salvage intensity-modulated radiotherapy with or without androgen deprivation after radical prostatectomy for rising or persisting prostate-specific antigen: 5-year results. Eur Urol 2011; 60: 842-849.

25. Goenka A, Magsanoc JM, Pei X et al. Improved toxicity profile following high-dose postprostatectomy salvage radiation therapy with intensity-modulated radiation therapy. Eur Urol 2011; 60: 1142-1148.

26. Macdonald OK, Schild SE, Vora SA et al. Salvage radiotherapy for palpable, locally recurrent prostate cancer after radical prostatectomy. Int J Radiat Oncol Biol Phys 2004; 58: 1530-1535.

27. Kukiełka AM, Strnad V, Stauffer P et al. Salvage brachytherapy in combination with interstitial hyperthermia for locally recurrent prostate carcinoma following external beam radiation therapy: a prospective phase II study. J Contemp Brachytherapy 2015; 7: 254-258.

28. Kunogi H, Wakumoto Y, Yamaguchi N et al. Focal partial salvage low-dose-rate brachytherapy for local recurrent prostate cancer after permanent prostate brachytherapy with a review of the literature. J Contemp Brachytherapy 2016; 3: 165-172.

29. Niehoff $\mathrm{P}$, Loch T, Nürnberg $\mathrm{N}$ et al. Feasibility and preliminary outcome of salvage combined HDR brachytherapy and external beam radiotherapy (EBRT) for local recurrences after radical prostatectomy. Brachytherapy 2005; 4: 141-145.

30. Strom TJ, Wilder RB, Fernandez DC. High-dose-rate brachytherapy with or without intensity modulated radiation therapy as salvage treatment for an isolated, gross local recurrence of prostate cancer post-prostatectomy. Brachytherapy $2014 ; 13:$ 123-127.

31. Losa A, Nava LD, Di Muzio N et al. Salvage brachytherapy for local recurrence after radical prostatectomy and subsequent external beam radiotherapy. Urology 2003; 62: 1068-1072.

32. Traudt K, Ciezki J, Klein EA. Low-dose-rate brachytherapy as salvage treatment of local prostate cancer recurrence after radical prostatectomy. Urology 2011; 77: 1416-1419.

33. Gomez-Veiga F, Mariño A, Alvarez L. Brachytherapy for the treatment of recurrent prostate cancer after radiotherapy or radical prostatectomy. BJU Int 2012; 109 Suppl 1: 17-21.

34. Gaztañaga M, Crook JM. Permanent seed brachytherapy for locally recurrent prostate cancer after radical prostatectomy: a case report and review of the literature. Brachytherapy 2013; 12: 338-342.

35. Kumar AMS, Smith KL, Reddy CA et al. Safety and efficacy of salvage low-dose-rate brachytherapy for prostate bed recurrences following radical prostatectomy. J Contemp Brachytherapy 2015; 7: 241-246.

36. Hosogoe S, Soma O, Matsumoto T et al. Salvage Brachytherapy for Castration-Resistant and External Beam RadiotherapyResistant Local Recurrence 17 Years after Radical Prostatectomy. Case Rep Urol 2015; 2015: 839738. 